\title{
Thermal radiation and entanglement in proton-proton collisions at energies available at the CERN Large Hadron Collider
}

\author{
O. K. Baker \\ Department of Physics, Yale University, New Haven, Connecticut 06520, USA \\ D. E. Kharzeev ${ }^{\dagger}$ \\ Department of Physics and Astronomy, Stony Brook University, New York 11794, USA \\ and Department of Physics and RIKEN-BNL Research Center, Brookhaven National Laboratory, \\ Upton, New York 11973, USA
}

(Received 27 June 2018; published 10 September 2018)

\begin{abstract}
The origin of the apparent thermalization in high-energy collisions is investigated using the data of the ATLAS and CMS Collaborations at the LHC. For this purpose, we analyze the transverse momentum distributions in the following proton-proton collision processes, all at $\sqrt{s}=13 \mathrm{TeV}$ : (i) inclusive inelastic $p p$ collisions; (ii) single- and double-diffractive Drell-Yan production $p p \rightarrow \mu^{+} \mu^{-} X$; and (iii) Higgs boson production. We confirm the relation between the effective temperature and the hard scattering scale observed at lower energies, and find that it extends even to the Higgs boson production process. In addition we find that the thermal component disappears in diffractive events (even though many charged hadrons are still produced). We discuss the implications of our study for the mechanism of multiparticle production-in particular, we test the hypothesis about the link between quantum entanglement and thermalization in high-energy collisions.
\end{abstract}

DOI: 10.1103/PhysRevD.98.054007

\section{INTRODUCTION}

Understanding the dynamics of multiparticle production in high energy collisions remains a big challenge for theory. This is because the description of real-time evolution of a strongly coupled non-Abelian gauge theory is notoriously difficult. Nevertheless, the availability of the diverse high quality data on multiparticle production from the experiments at the LHC and RHIC should allow us to motivate and inform the theory. In this work, we address the origin of the apparent thermalization in high-energy collisions that is usually inferred from the presence of the exponential component in the transverse momentum distributions of produced particles and the thermal abundances of the hadron yields (see [1] for a review). The emergence of the thermal features in a high energy proton-proton collision is surprising, as the number of secondary interactions in this process is relatively low and does not favor

\footnotetext{
*oliver.baker@yale.edu

†dmitri.kharzeev@stonybrook.edu
}

Published by the American Physical Society under the terms of the Creative Commons Attribution 4.0 International license. Further distribution of this work must maintain attribution to the author(s) and the published article's title, journal citation, and DOI. Funded by SCOAP ${ }^{3}$. thermalization through conventional final-state interaction mechanisms.

In this paper we will investigate the possibility that the apparent thermalization in high energy collisions is achieved during the rapid "quench" induced by the collision due to the high degree of entanglement inside the wave functions of the colliding protons [2]. An example of such a "quantum thermalization through entanglement" is a recent experimental study of a quench in the Bose-Einstein condensate of $\mathrm{Rb}$ atoms where the entanglement was found to induce a rapid eigenstate thermalization [3]. Theoretical studies of quenches in entangled quantum systems described by $(1+1)$-dimensional conformal field theories [4,5] indicate that at late times the system can be described by a generalized thermal Gibbs ensemble with an effective temperature set by the energy cutoff for the ultraviolet modes.

Since a high-energy collision can be viewed as a rapid quench of the entangled partonic state [2], it is thus possible that the effective temperature inferred from the transverse momentum distributions of the secondaries in a collision can depend upon the momentum transfer, that is an ultraviolet cutoff on the quantum modes resolved by the collision. In analyzing the high-energy collisions with different characteristic momentum transfer $Q$ we thus expect to find different effective temperatures $T \sim Q$. We can also look at the inelastic events characterized by a rapidity gap, where the proton is probed as a whole, and no entanglement entropy 
arises [2] - in this case, if the quantum entanglement is responsible for the thermalization, we expect no thermal radiation.

In fact, it has been observed [6] in deep-inelastic scattering at HERA that while the thermal component of hadron spectra is a prominent feature of inclusive events, this thermal radiation disappears in the events characterized by the rapidity gap. Since diffractive processes with a rapidity gap involve the entire wave function of the proton, there is no associated entanglement entropy-so this observation hints at a link between the entanglement and thermalization. The relation between the effective temperature and the saturation momentum (that is an UV cutoff on the gluon modes in an inclusive interaction) that has been deduced [7] from the inclusive data on inelastic collisions at RHIC energies also agrees with this hypothesis. An alternative view is that the thermal radiation possesses a universal effective temperature $T \sim \Lambda$, where $\Lambda$ is the $\mathrm{QCD}$ scale that determines the mass gap in this theory.

We would like to emphasize that the existence of a universal effective temperature deduced from the hadron abundances does not contradict the growth of the effective temperature as deduced from the transverse momentum spectra. This is because the effective temperature deduced from the hadron abundances reflects the property of confinement, and is determined through dimensional transmutation of QCD, by the QCD scale $\Lambda$. In the oldfashioned, but definitely correct, approach proposed by Hagedorn [8], this limiting temperature is determined by the density of hadron states, which in turn is related to the QCD string tension, and thus to $\Lambda$.

The temperature deduced from the transverse momentum spectra, on the other hand, reflects thermalization at the quark-gluon level. This kind of an effective temperature is not limited from above and can indefinitely increase with the collision energy. Because of the energy-momentum conservation during the hadronization, the growth of the temperature of quarks and gluons leads to the hardening of the hadron transverse momentum distributions. In fact it has been known for some time that the phase space distributions of produced hadrons closely reflect the phase space distributions of partons computed in QCD-this property is known as the local parton-hadron duality [9].

The large amount of data accumulated by the LHC experiments should allow to disentangle these distinct possibilities, and we will attempt to do it in this paper. Specifically, we perform the comparison of the transverse momentum distributions in the following proton-proton collision processes at $\sqrt{s}=13 \mathrm{TeV}$ : (i) inclusive inelastic $p p$ collisions; (ii) single- and double-diffractive Drell-Yan production $p p \rightarrow \mu^{+} \mu^{-} X$; and (iii) Higgs boson production.

The paper is organized as follows. In Sec. II we briefly summarize the theoretical ideas on the role of entanglement in high energy collisions, and discuss the possible link between entanglement and thermalization. In Sec. III we analyze the transverse momentum distributions of charged hadrons produced in inelastic $p p$ collisions at $\sqrt{s}=$ $13 \mathrm{TeV}$. Here we find both the "thermal" (falling off exponentially) and "hard" (falling off as a power) components, with the effective temperature and semi-hard scattering scales related in a way similar to what has been found at lower energies [7]. In Sec. IV we analyze the single- and double-diffractive Drell-Yan production $p p \rightarrow \mu^{+} \mu^{-} X$ at $\sqrt{s}=13 \mathrm{TeV}$. The dominant mechanism of $\mu^{+} \mu^{-}$production is the photon-photon fusion $\gamma \gamma \rightarrow \mu^{+} \mu^{-}$, so these diffractive processes allow an analysis of the fragmentation of a high energy proton in an intense electromagnetic field produced by the other proton. We observe that the thermal component of the hadron spectrum disappears in this class of events even though the events are inelastic and do produce many hadrons. In Sec. V we analyze the transverse momentum distribution of the Higgs bosons produced in $p p$ collisions at $\sqrt{s}=13 \mathrm{TeV}$. Surprisingly, we find that this transverse momentum distribution is also accurately described by the superposition of a power-like "hard" component with the hardness scale set by the Higgs boson mass $M_{H}$ and the thermal component with a very high effective temperature $T \sim M_{H}$, where the proportionality coefficient is close to the one observed for other inelastic processes. Finally, in Sec. VI we discuss our results and their implications for understanding the mechanism of apparent thermalization in high energy collisions.

\section{ENTANGLEMENT AND THERMALIZATION IN HIGH ENERGY COLLISIONS}

Recently, it has been proposed that quantum entanglement is at the origin of parton distributions measured in hard processes [2]. Let us briefly summarize these arguments here. A hard process probes only the part of the proton wave function that is localized in a region of space that we denote $A$. For a hard process with a momentum transfer $q^{2}=-Q^{2}$ and Bjorken variable $x$, this region has a transverse size $\sim 1 / Q$ and, in the proton's rest frame, longitudinal size $\sim(m x)^{-1}$, where $m$ is the proton mass.

Let us denote by $B$ the region of space complementary to $A$, so that the entire space is $A \cup B$. The physical states inside the region $A$ probed by the hard process are states in a Hilbert space $\mathcal{H}_{A}$ of dimension $n_{A}$, and unobserved states in the region $B$ belong to the Hilbert space $\mathcal{H}_{B}$ of dimension $n_{B}$. The composite system in $A \cup B$ (the entire proton) is then described by the vector $\left|\Psi_{A B}\right\rangle$ in the space $\mathcal{H}_{A} \otimes \mathcal{H}_{B}$ that is a tensor product of the two spaces:

$$
\left|\Psi_{A B}\right\rangle=\sum_{i, j} c_{i j}\left|\varphi_{i}^{A}\right\rangle \otimes\left|\varphi_{j}^{B}\right\rangle,
$$

where $c_{i j}$ are the elements of the matrix $C$ that has a dimension $n_{A} \times n_{B}$. If one can find such states $\left|\varphi^{A}\right\rangle$ and $\left|\varphi^{B}\right\rangle$ that $\left|\Psi_{A B}\right\rangle=\left|\varphi^{A}\right\rangle \otimes\left|\varphi^{B}\right\rangle$, i.e., that the sum (1) 
contains only one term, then the state $\left|\Psi_{A B}\right\rangle$ is separable, or a product state. Otherwise the state $\left|\Psi_{A B}\right\rangle$ is entangled.

The Schmidt decomposition theorem states that the pure wave function $\left|\Psi_{A B}\right\rangle$ of our bi-partite system can be expanded as a single sum

$$
\left|\Psi_{A B}\right\rangle=\sum_{n} \alpha_{n}\left|\Psi_{n}^{A}\right\rangle\left|\Psi_{n}^{B}\right\rangle
$$

for a suitably chosen orthonormal sets of states $\left|\Psi_{n}^{A}\right\rangle$ and $\left|\Psi_{n}^{B}\right\rangle$ localized in the domains $A$ and $B$, respectively, where $\alpha_{n}$ are positive and real numbers that are the square roots of the eigenvalues of matrix $C C^{\dagger}$. In the parton model, we assume that this full orthonormal set of states is given by the Fock states with different numbers $n$ of partons.

The density matrix of the mixed state probed in region $A$ can now be written down as

$$
\rho_{A}=\operatorname{tr}_{B} \rho_{A B}=\sum_{n} \alpha_{n}^{2}\left|\Psi_{n}^{A}\right\rangle\left\langle\Psi_{n}^{A}\right|,
$$

where $\alpha_{n}^{2} \equiv p_{n}$ is the probability of a state with $n$ partons. The identification of the basis $\left|\Psi_{n}^{A}\right\rangle$ in the Schmidt decomposition (2) with the states with a fixed number $n$ of partons is natural-only in this case we do not have to deal with quantum interference between states with different numbers of partons, as such interference is absent in the parton model. Because the parton model represents a description of QCD that is a relativistic field theory, the number of terms in the sum (2) (the Schmidt rank) is in general infinite. Note that a pure product state with no entanglement would have a Schmidt rank one.

The von Neumann entropy of this state is given by

$$
S=-\sum_{n} p_{n} \ln p_{n}
$$

This entropy results from the entanglement between the regions $A$ and $B$, and can thus be interpreted as the entanglement entropy. In terms of information theory, Eq. (4) represents the Shannon entropy for the probability distribution $\left(p_{1}, \ldots, p_{N}\right)$. The QCD evolution equations can be used to evaluate the probabilities $p_{n}$, and thus the entanglement entropy (4).

After the hard scattering takes place, the mixed quantum state characterized by the entanglement entropy (4) undergoes the evolution toward the final asymptotic state of hadrons measured in the detectors. This final state is characterized by the Boltzmann entropy; how does this entropy relate to the initial entanglement entropy of the system? Does the produced Boltzmann entropy correspond to an entropy of a thermal ensemble?

To address these questions, let us consider the protonproton collision in the reference frame where one of the protons is at rest. As discussed above, in this frame the partonic configuration of the high-momentum proton is prepared long before the collision, at a distance $\sim(m x)^{-1}$.
The proton itself is an eigenstate $\left|\psi_{0}\right\rangle$ of the QCD Hamiltonian $H_{0}$. When the collision takes place, this configuration undergoes a rapid "quench," and evolves according to a new Hamiltonian $H=H_{0}+V(t)$ where $V(t)$ is the term induced by the inelastic interaction. Since an inelastic interaction in QCD is induced by the gluon exchange, the term $V(t)$ represents an effect of the pulse of the color field. The onset of this pulse in a hard scattering with a hardness scale $Q$, by the uncertainty principle, is $\tau \sim 1 / Q$ (we write it in the comoving frame, so $\tau$ is the proper time). Since this time is short on the QCD scale, $\tau \ll 1 / \Lambda$, the quench creates a highly excited multi-particle state.

For the case of a short pulse of (chromo) electric field, the produced particles have thermal-like exponential spectra with an effective temperature of $T \simeq(2 \pi \tau)^{-1} \simeq Q /(2 \pi)$ [10]. The derivation in [10] involved a semiclassical approximation, but the same result holds for specific time profiles of the pulse when exact solutions can be found [11]. The thermal spectrum in this case can be attributed to the emergence of an event horizon formed due to the acceleration induced by the electric field $[10,12]$.

These arguments point to the proportionality between the momentum scale $Q$ in an inelastic interaction and the effective temperature $T$ inferred from the transverse momentum distributions $[7,10,13]$ :

$$
T=c \frac{Q}{2 \pi},
$$

where $c$ is a universal (energy-independent) coefficient of order one. In an inclusive inelastic event, the scale $Q$ has to be identified $[7,10,13]$ with the "saturation momentum" $Q_{s}$ [14-16] that depends on the Bjorken $x$ and thus on the energy of the collision and the rapidity at which the measurement of the spectra is performed. In a hard process, the scale $Q$ is set by the kinematics of the process.

The emergence of thermal behavior in an entangled quantum system undergoing a quench has been recently observed in Bose-Einstein condensate of Rb atoms [3]. The effective temperature was found to depend on the properties of the quench, similarly to the situation discussed above. The studies of entanglement and thermalization using an ensemble of trapped atomic ions were also performed in [17]. Here the authors show experimental evidence for quantum entanglement and information propagation, results that can be compared to measurements of thermalization in these systems $[18,19]$.

Is it possible to predict the amount of produced Boltzmann entropy if one knows the initial entanglement entropy? In the case of a high energy collision, this would allow us to predict the produced entropy if the parton distributions (interpreted in terms of entanglement entropy [2]) are known. The comparison to the LHC data on hadron multiplicity distributions performed in [2] indicates that the produced Boltzmann entropy is quite close to the initial entanglement entropy. 
Unfortunately, very little is known at present on general grounds about the transformation of the entanglement entropy into the Boltzmann entropy following the quench. This problem is important and emerges in many areas of physics-for example, solving it would enable understanding of qubit decoherence in quantum computers. The theoretical results available at present are mostly limited to the case of conformal field theory (CFT). In particular, it is known $[4,5]$ that for a rapid quench (such as the one that occurs in a high-energy collision) in a $(1+1)$ dimensional CFT the entanglement entropy of a segment of length $L$ first grows linearly in time, until $t \simeq L / 2$, and then saturates at the value

$$
S(t) \simeq \frac{c}{3} \ln \tau_{0}+\frac{\pi c L}{12 \tau_{0}},
$$

where $c$ is the conformal charge of the CFT, and $\tau_{0}^{-1}$ is the energy cutoff for the ultraviolet modes. Comparing this to the entropy of a thermal $(1+1)$ dimensional system at a temperature $T, S_{\text {therm }} \simeq \frac{c}{3} L T$, we infer that the effective temperature is $T \sim \tau_{0}^{-1}$. Drawing an analogy to our case of a $(3+1)$ dimensional hard collision, we identify $Q=\tau_{0}^{-1}$, and expect to find an effective temperature $T \sim Q$, in accord with our previous arguments.

The interpretation of the result (6) is the following $[4,5,20]$. The quench leads to the production of entangled (quasi)particle pairs, since what used to be the ground state of the undisturbed Hamiltonian $H_{0}$ is a highly excited state of the Hamiltonian after the quench, $H=H_{0}+V(t)$. The entangled pairs produced by the quench propagate along the light cone, and contribute to the entanglement entropy of the segment of length $L$ if only one particle of the pair is detected within this segment. Shortly after the quench, only particle pairs produced near the boundary of the segment thus contribute to the entanglement, and the entanglement entropy is not extensive in the length $L$. However, at times $t>L / 2$, even in the center of the segment one can detect a particle whose entangled partner is outside of the segment-this means that the entanglement entropy receives contributions from the entire segment, and should scale extensively in $L$ in accord with the result (6). This scaling is a necessary condition for an effective thermalization.

For a quench induced by a high-energy collision, we sketch the resulting picture of thermalization from entanglement in Fig. 1. Note that the hardest quasiparticle modes that propagate along the light cone thermalize first. For the softer particles that propagate in the interior of the light cone, it takes a longer time to thermalize, i.e., to exhibit an extensive scaling of the entropy. The detection of particles is assumed to be performed within the interval of length $L$ [see Eq. (6)], corresponding to a limited range in (pseudo) rapidity. While Eq. (6) has been obtained in the framework of CFT, the simple physical interpretation of this result makes its broader validity quite likely.

It is instructive to point out the difference in the mechanisms of thermalization expected at weak and strong

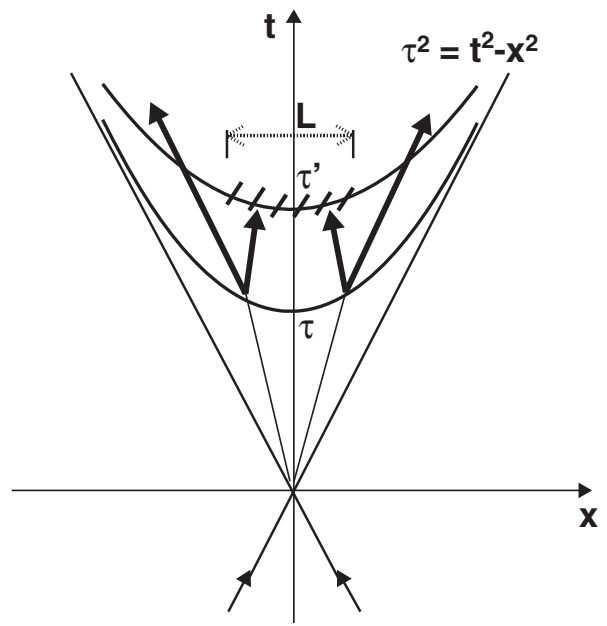

FIG. 1. A sketch illustrating quantum thermalization through entanglement in a high energy collision. The entangled particle pairs produced at a proper time $\tau$ contribute to the entanglement entropy in the interval of length $L$ shown by the hashed segment of the curve at a proper time $\tau^{\prime}>\tau$.

coupling. At weak coupling, the "bottom-up" thermalization mechanism [21] also yields an effective temperature $T \sim Q_{s}$ in inelastic high energy collisions. However the thermalization in this picture begins from the soft, lowmomentum modes that eventually draw the energy from the harder modes; the thermalization of the hard, highmomentum modes is thus expected to take a parametrically long time proportional to the inverse power of the (small) coupling constant [21]. On the other hand, in strongly coupled entangled systems the process of thermalization is fast and determined by the size of the system and the parameters of the quench; moreover, it starts from the hardest modes resolved in the process. In the dual holographic description of conformal field theory, this process is described by the formation of trapped surface near the Minkowski boundary that then falls into the AdS bulk, corresponding to the spreading of thermalization from hard to soft modes [22,23]. A similar picture emerges from the analysis of entanglement entropy in an expanding string [24], where the entropy has been found to have a thermal form with an effective temperature $T \sim 1 / \tau$ at early time $\tau$.

The arguments presented above are qualitative at best, and can definitely be questioned. Nevertheless, we feel that they provide enough motivation to look into the structure of inelastic collision events at high energies, and to explore the possible relation between the effective temperature and the hard scale of the collision. We will now proceed to performing such an analysis.

\section{CHARGED HADRON TRANSVERSE MOMENTUM DISTRIBUTION}

Data from proton-proton collisions at $13 \mathrm{TeV}$ center of mass energy yielding multiple charged particles in the final state have been recorded by the ATLAS collaboration at 


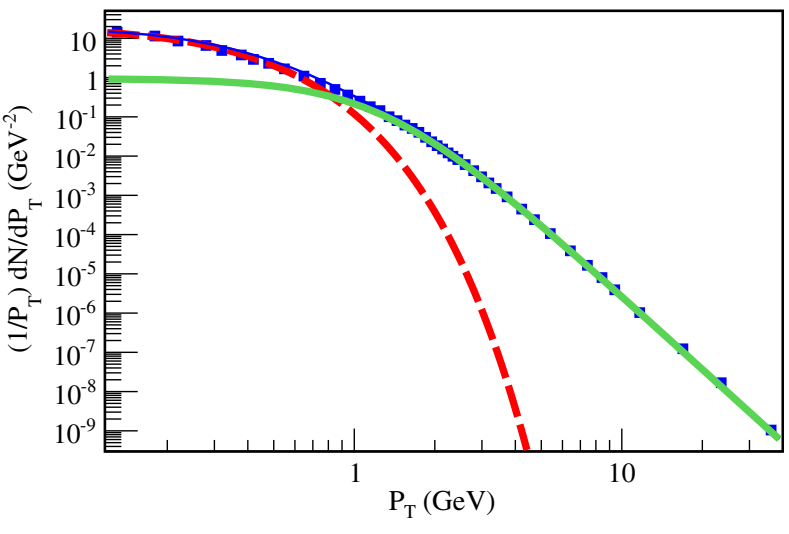

FIG. 2. Transverse momentum distribution of charged hadrons in proton-proton collisions at $\sqrt{s}=13 \mathrm{TeV}$. The curves shown are exponential (red dashed) and power law (green solid) corresponding to the thermal and hard scattering contributions respectively, and the sum of these two contributions (blue, thin solid).

CERN's LHC in 2016 [25]. The data set corresponds to an integrated luminosity of $151 \mu \mathrm{b}^{-1}$ for charged particles that have transverse momenta greater than $100 \mathrm{MeV} / \mathrm{c}$ and absolute pseudorapidity of less than 2.5. Events that contain two or more charged particles in the final state were selected for analysis. In order to remove the presence of strangeness or heavier flavor charged particles from the sample, final state hadrons that originate in the primary $\mathrm{pp}$ interaction and that have a lifetime of greater than $30 \mathrm{ps}$ were excluded from the final selected events. Additionally, secondary charged particles that are the result of particle decays from this data set that have a lifetime greater than $30 \mathrm{ps}$ were also excluded. The data set effectively excluded charged strange baryons from the results used in the present analysis. Comparisons with event generators PYTHIA 8 and EPOS (LHC tune) indicate that the majority of selected events are nondiffractive and that the process is dominated by t-channel gluon exchanges. Transverse momentum bin widths from $0.1 \mathrm{GeV}$ at low $P_{T}$ to as large as $20 \mathrm{GeV}$ at higher $P_{T}$ were used in the analysis [25].

The normalized charged hadron transverse momentum distribution is shown in Fig. 2. The thermal component is shown by the exponential, red dashed curve; we parametrize it as

$$
\frac{1}{N_{e v}} \frac{1}{2 \pi P_{T}} \frac{d^{2} N_{e v}}{d \eta d P_{T}} \sim \mathrm{A}_{\text {therm }} \exp \left(-\mathrm{m}_{\mathrm{T}} / \mathrm{T}_{\text {th }}\right),
$$

where the transverse mass $m_{T}$ is given by $m_{T} \equiv \sqrt{m^{2}+P_{T}^{2}}$ ( $m$ is the hadron mass; we assume that the spectrum is dominated by pions), and $T_{t h}$ is an effective temperature. The hard scattering (power law, green solid curve) component is parametrized as in [7],

$$
\frac{1}{N_{e v}} \frac{1}{2 \pi P_{T}} \frac{d^{2} N_{e v}}{d \eta d P_{T}} \sim \frac{\mathrm{A}_{\text {hard }}}{\left(1+\frac{\mathrm{m}_{\mathrm{T}}^{2}}{\mathrm{~T}^{2} \mathrm{n}}\right)^{\mathrm{n}}},
$$

where $T$ and $n$ are parameters to be determined from the fit. The sum of the two terms is shown by the blue solid curve.

The value $\mathrm{T}_{t h}=0.17 \mathrm{GeV}$ describes well the experimental transverse momentum distribution; it agrees with that expected from the extrapolation of the relation [7] deduced at lower energies

$$
\mathrm{T}_{t h}=0.098 \cdot\left(\sqrt{\mathrm{s} / \mathrm{s}_{0}}\right)^{0.06} \mathrm{GeV}
$$

to the LHC energy of $\sqrt{s}=13 \mathrm{TeV} ; s_{0}=1 \mathrm{GeV}^{2}$. Similarly, the hard scale parameter T extracted in [7] is

$$
\mathrm{T}=0.409 \cdot\left(\sqrt{\mathrm{s} / \mathrm{s}_{0}}\right)^{0.06} \mathrm{GeV} .
$$

Note that the parametrizations (9) and (10) imply that the effective temperature $\mathrm{T}_{t h}$ is proportional to the hard scale $\mathrm{T}$, in accord with our discussion in Sec. II.

Our fit to the charged hadron transverse momentum distribution yields the hard scale parameter $T=0.72 \mathrm{GeV}$ and $n=3.1$. This value of $T$ is in agreement with the extrapolation of (10) to $\sqrt{s}=13 \mathrm{TeV}$, but the value of $n$ is smaller, reflecting the slower fall-off of the transverse momentum distribution at the LHC energy.

Let us define the ratio $R$ of the integral under the power law (hard scattering) curve and the integral under the total (hard scattering plus thermal component) curve of the fit in Fig. 2:

$$
\mathrm{R}=\frac{\text { power }}{\text { power }+ \text { exponential }} .
$$

We find for it the value of $R \simeq 0.16$, in agreement with the ratio calculated from the charged hadron spectra in inelastic proton-proton collisions at ISR energies of $\sqrt{s}=23,31$, 45 , and $53 \mathrm{GeV}[6]$.

\section{DIMUON PAIR TRANSVERSE MOMENTUM DISTRIBUTION FROM $\gamma \gamma$ SCATTERING IN PROTON-PROTON COLLISIONS}

Proton-proton ( $p p$ ) collisions at the LHC often proceed through the photon-photon $(\gamma \gamma)$ interactions. In this case, the final state of the collision contains the protons, or the products $X^{\prime}, X^{\prime \prime}$ of their diffractive dissociation. The ATLAS collaboration made measurements of the reaction

$$
p p(\gamma \gamma) \rightarrow \mu^{+} \mu^{-} X^{\prime} X^{\prime \prime}
$$

at $13 \mathrm{TeV}$ center of mass energy in pp collisions [26]. The relevant Drell-Yan (DY) production processes are exclusive production (with two intact protons in the final state), single diffraction (in which one of the incident protons dissociates into an inelastic state), and double diffraction (in which both of the incident protons dissociate). Selection of the exclusive $\gamma \gamma \rightarrow \mu^{+} \mu^{-}$process was implemented by only 


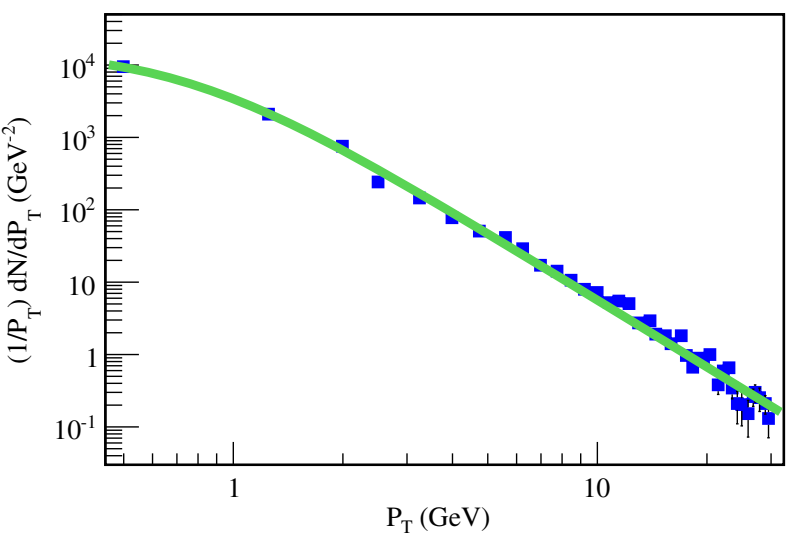

FIG. 3. Transverse momentum distribution of normalized event distribution $\frac{1}{P_{T}} \frac{d N_{\mu \mu}}{d P_{T}}$ in units of $\mathrm{GeV}^{-2}$ versus the transverse momentum muon pair transverse momentum in units of $\mathrm{GeV}$ for the $(\mathrm{pp})(\gamma \gamma) \rightarrow \mu^{+} \mu^{-}(\mathrm{pp})$ reaction. The curve shown (green, solid) is the power law contribution corresponding to the hardscattering process.

including events that have both a $\mu^{+}$and $\mu^{-}$track while excluding events that show additional charged particle activity with transverse momenta greater than $400 \mathrm{MeV}$ and within the pseudorapidity range considered here. DY and multijet contributions, which are backgrounds to the exclusive reaction, are vetoed with these cuts. Additional DY vetoing is achieved by excluding events that yield a dimuon invariant mass greater than $70 \mathrm{GeV}$. The analysis performed in [26] shows that at the transverse momenta of the Drell-Yan pair below $1.5 \mathrm{GeV}$ the DY production is dominated by the exclusive process, whereas at larger transverse momenta the single and double diffractive processes with inelastic final states dominate.

In the most recent ATLAS analysis of the reaction (12) care was taken to select diffractive events that proceed through the $\gamma \gamma$ scattering. As argued in [2,7] such diffractive events are expected to have a suppressed thermal (exponential) component. This is because in these diffractive processes the photon interacts coherently with the entire proton, and no entanglement entropy arises, as discussed in Sec. II. As the presence of the thermal component in this approach is the consequence of the entanglement, we expect it to be absent in diffractive events.

Figure 3 shows the transverse momentum distribution in the case of $\gamma \gamma$ production of di-muon pairs in proton-proton collisions at $13 \mathrm{TeV}$ center of mass energy; the transverse momentum bin widths of $1.3 \mathrm{GeV}$ were used in [26]. As can be seen from Fig. 3, the hard scattering term alone describes well the distribution, and there is no thermal (exponential) component visible in the distribution. The ratio $R$ defined in the previous section in this case is $R \simeq 1$, in agreement with our theoretical expectations and the previous data for $\gamma \gamma$ scattering at OPAL at $\sqrt{s}=15$ and $35 \mathrm{GeV}$ that also show no thermal component, with $R$ close to one.

\section{HIGGS BOSON TRANSVERSE MOMENTUM DISTRIBUTION}

With the newest (and final) member of the standard model now discovered, we can investigate whether the transverse momentum distribution of the Higgs boson is affected by the thermalization processes. While there is little doubt that the integrated cross sections of the Higgs production in general are adequately described by perturbation theory (see [27] for a review), it is possible that the QCD radiation in this process, and thus the Higgs boson transverse momentum distributions, are affected by the entanglement.

The Higgs boson transverse momentum distributions have been measured by both ATLAS and CMS collaborations in the discovery mode channels: Higgs boson decays to four leptons (electrons and muons) [28] and Higgs boson decays to $\gamma \gamma[29,30]$. The data considered here are for proton-proton collisions at $\sqrt{s}=13 \mathrm{TeV}$ center of mass energy collected during Run 2 in 2015 and 2016.

\section{A. Higgs boson decay to $\gamma \gamma$}

The dominant particle level process in the reaction $\mathrm{pp} \rightarrow$ $\mathrm{H} \rightarrow \gamma \gamma$ is gluon-gluon fusion, followed by the relatively less frequent vector boson fusion (VBF), associated production with top quarks ( $\mathrm{ttH}$ ) and associated production with a vector boson $(\mathrm{VH})$. The fiducial cross sections for the reactions considered here are defined as the two photon final states where the photons are well isolated and are restricted to the absolute pseudorapidity region $|\eta| \leq 2.37$, and where the leading and subleading photons satisfy the requirement that the transverse momentum-diphoton invariant mass ratio $P_{T} / m_{\gamma \gamma}$ is greater than 0.35 and 0.25 , respectively. Photons must have a transverse momentum greater than the threshold of $25 \mathrm{GeV}$, and only photons that are detected outside of the ATLAS detector crack region, $1.37 \leq \eta \leq 1.52$ in pseudorapidity are retained. The diphoton invariant mass for Higgs boson reconstruction is restricted to an invariant mass range between $105 \mathrm{GeV}$ and $160 \mathrm{GeV}$, inclusive.

Since the fiducial volumes of both ATLAS and CMS analyses are not too different given the uncertainties in the measurements, the results from both experiments are included in the current analysis. In Fig. 4 the transverse momentum distribution of the Higgs bosons is shown in the range from $8 \mathrm{GeV}$ to $390 \mathrm{GeV}$ for combined ATLAS and CMS data. As can be seen from Fig. 4, there clearly are both the hard scattering (power law) and thermal (exponential) components in the transverse momentum distribution, similarly to the case explored in Sec. III. In fact, due to the much larger range of the available transverse momenta, the separation between the hard and thermal components is even more defined.

The power-law and exponential distributions yield an effective temperature $T_{t h} \simeq 3.5 \mathrm{GeV}$ and the hard scale 


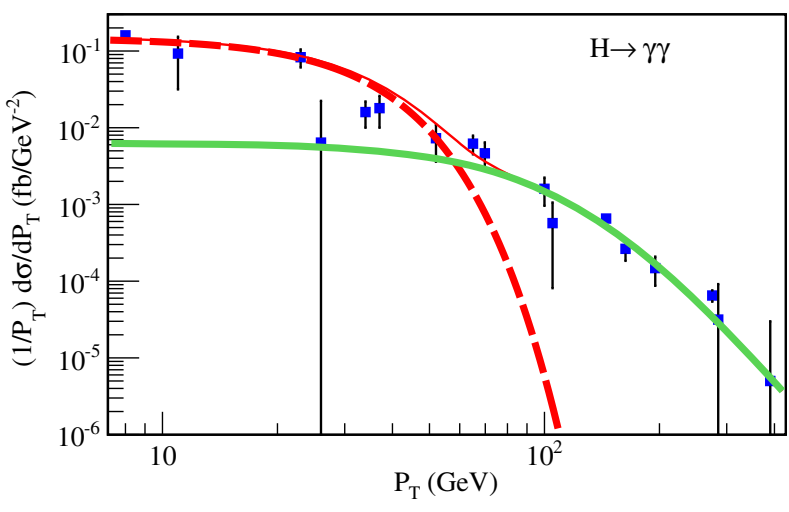

FIG. 4. Transverse momentum distribution of the Higgs bosons reconstructed from the $H \rightarrow \gamma \gamma$ decay in proton-proton collisions at $\sqrt{s}=13 \mathrm{TeV}$. The curves shown are exponential (red dashed) and power law (green, solid) components corresponding to thermal and hard scattering contributions respectively; the sum of the two contributions is shown by blue, thin solid curve.

parameter $T \simeq 14.4 \mathrm{GeV}$ that are about 20 times larger than the values derived from the charged hadron data in Sec. III. Interestingly, the ratio $R$ defined by (11) and extracted from Fig. 4 is $R=0.15 \pm 0.05$ that is very close to the one determined from the charged hadron distribution in protonproton collisions studied in Sec. III, $R=0.16 \pm 0.05$.

\section{B. Higgs boson decay to four leptons}

The Higgs boson decays to the additional high resolution final state channel (four leptons) were used to extract the transverse momentum $\left(P_{T}\right)$ distributions in both ATLAS [29] and CMS [30]. Muon (electron) identification requirements are transverse momentum thresholds of 5 (7) GeV and an absolute pseudorapidity window of $|\eta|$ less than 2.7 (2.54) in ATLAS. Systematic uncertainties on the detector correction and acceptance factors are at most $3.2 \%$ and are mostly less than $1 \%$.

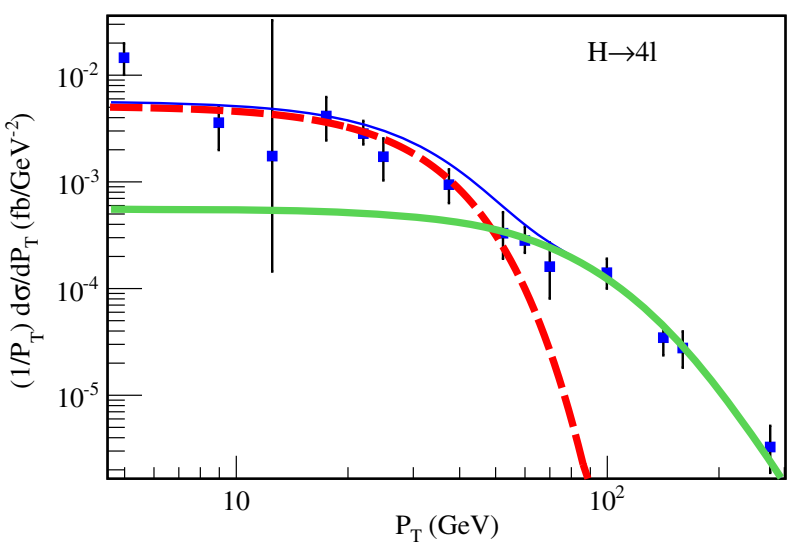

FIG. 5. The differential cross section of the Higgs boson production reconstructed from the $\mathrm{H} \rightarrow 4 l$ (electrons, muons) decay in proton-proton collisions at $\sqrt{s}=13 \mathrm{TeV}$. The curves shown are defined as before.
TABLE I. The effective temperature $T_{t h}$, the hard scale parameter $T$, and the fraction of the hard component in the spectrum (11) for different processes.

\begin{tabular}{lccc}
\hline \hline $\mathrm{T}_{\text {th }}, \mathrm{GeV}$ & $\mathrm{T}, \mathrm{GeV}$ & $\mathrm{R}$ & Process \\
\hline $0.17 \pm 0.03$ & $0.72 \pm 0.1$ & $0.16 \pm 0.05$ & $\mathrm{pp} \rightarrow$ charged hadrons \\
None & $0.1 \pm 0.02$ & $1.0 \pm 0.1$ & $\mathrm{pp}(\gamma \gamma) \rightarrow(\mu \mu) \mathrm{pp}$ \\
$3.5 \pm 0.7$ & $14.4 \pm 0.3$ & $0.15 \pm 0.05$ & $\mathrm{pp} \rightarrow \mathrm{H} \rightarrow \gamma \gamma$ \\
$3.5 \pm 0.7$ & $14.4 \pm 0.3$ & $0.23 \pm 0.05$ & $\mathrm{pp} \rightarrow \mathrm{H} \rightarrow 4 \mathrm{l}(\mathrm{e}, \mu)$ \\
\hline \hline
\end{tabular}

Shown in Fig. 5 is the normalized differential cross section, $\frac{1}{p^{T}} \frac{d \sigma}{d p^{T}}$ in units of $\mathrm{fb} / \mathrm{GeV}^{2}$ between 5 and $275 \mathrm{GeV}$ transverse momenta for the $H \rightarrow Z Z^{*} \rightarrow 4 l$ reaction. Just as for the case $H \rightarrow \gamma \gamma$ in the previous subsection, there is here also a clear hard scattering component as well as a thermal component to the full distribution. (The curves are defined as before). As in the $H \rightarrow \gamma \gamma$ distribution described in the previous subsection, the power-law and exponential components yield an effective temperature and the hard scale parameter that are about 20 times larger than those determined from the charged hadron spectrum. The ratio $\mathrm{R}$ calculated in the 41 case [see Eq. (11)] is $R=0.23 \pm 0.05$, which is consistent within the error bars with the value $R=0.15 \pm 0.05$ extracted from the $H \rightarrow \gamma \gamma$ decay mode.

Table I presents a compilation of the effective temperatures, hard scale parameters and the ratio $\mathrm{R}$ [defined by (11)] for the processes considered in this paper.

We have found that the shape of the Higgs transverse momentum distribution in the entire $p_{T}$ range is consistent with the "thermal + power" shape that we assume for all other processes. The effective temperature of the thermal distribution is determined by the Higgs mass and is thus very large, about $3.5 \mathrm{GeV}$. In the framework of the standard approach, there is no reason to expect that such a description would apply-but the data indicate that it does. This is consistent with the assumption that the effective temperature is proportional to the hard scale of the process that we apply throughout the paper.

\section{DISCUSSION}

The theoretical arguments and the analysis of the LHC data presented above point to an unconventional mechanism of apparent thermalization in high-energy collisions. The effective temperature $T_{t h}$ deduced from the data has been found here to be nonuniversal and proportional to the hard scale of the collision $T$, i.e., to the momentum transfer, with $T \simeq 4.2 T_{t h}$. Our analysis at the LHC energy confirms a similar conclusion made at lower energies [7]. Strikingly, this conclusion seems to apply even to the Higgs boson production, suggesting that even in this very hard process the QCD radiation may be affected by thermalization. This is demonstrated for the first time. Moreover, we have found that the thermal component of the spectrum is entirely absent in diffractive production at $13 \mathrm{TeV}$ c.m.e. (even though many 
hadrons are still produced in this case) — this again points to the nonuniversal, process-dependent, nature of thermalization. The data sets used here are the ones with sufficiently small statistical and systematic uncertainties to enable stringent comparisons between experimental results and theoretical predictions at the highest LHC energies.

All of these features of the data seem to be consistent with the picture of thermalization induced by quantum entanglement. Indeed, in this scenario the effective temperature is proportional to the momentum transfer $Q$ in the collision that provides the UV cutoff for the quantum modes. This expectation agrees with our analysis of the inclusive charged hadron and Higgs boson transverse momentum distributions, in which the typical momentum transfers are vastly different. We have found that the thermal component is present in both cases, but the values of the effective temperature differ by over an order of magnitude [31]. In diffractive production, one studies the coherent response of the entire proton, and there is no associated entanglement entropy [2]. In this case, in the "thermalization through entanglement" picture advocated here, we expect to find no thermal component at all. This prediction is confirmed by the data on diffractive Drell-Yan production analyzed in this paper, as well as by the diffractive deep-inelastic scattering data [6].

These findings suggest a deep connection between quantum entanglement and thermalization in high-energy hadron collisions that has to be investigated further. On the experimental side, our study can be extended in several directions. In deep inelastic scattering at the future Electron Ion Collider, it would be necessary to combine the measurements of the structure functions with the study of hadronic final states, especially in the target fragmentation region. In proton-proton, proton-nucleus and nucleusnucleus collisions at RHIC and LHC one can study the thermal component and the corresponding effective temperature in hard processes characterized by different momentum transfer. It would also be very interesting to investigate the dependence of the apparent thermalization on rapidity - the picture presented in Fig. 1 suggests that thermalization is achieved faster if we perform a measurement in a smaller rapidity interval.

It is clear that we are still very far from understanding thermalization in high-energy QCD, and much remains to be done both in theory and in experiment. Nevertheless, basing on the arguments and analysis presented above we believe that "thermalization through entanglement" emerges as a promising research direction that has to be pursued further.

\section{ACKNOWLEDGMENTS}

The authors acknowledge support from the U.S. Department of Energy, Award No. E00176 (O. K. B.) and Contracts No. DE-FG-88ER40388 and DE-AC0298CH10886 (D. E. K.).
[1] F. Becattini, An introduction to the statistical hadronization model, arXiv:0901.3643.

[2] D. E. Kharzeev and E. M. Levin, Deep inelastic scattering as a probe of entanglement, Phys. Rev. D 95, 114008 (2017).

[3] A. M. Kaufman, M. E. Tai, A. Lukin, M. Rispoli, R. Schittko, P. M. Preiss, and M. Greiner, Quantum thermalization through entanglement in an isolated many-body system, Science 353, 794 (2016).

[4] P. Calabrese and J. Cardy, Evolution of entanglement entropy in one-dimensional systems, J. Stat. Mech. (2005) P04010.

[5] P. Calabrese and J. Cardy, Quantum quenches in $1+1$ dimensional conformal field theories, J. Stat. Mech. (2016) 064003.

[6] A. A. Bylinkin and A. A. Rostovtsev, Role of quarks in hadroproduction in high energy collisions, Nucl. Phys. B888, 65 (2014).

[7] A. A. Bylinkin, D. E. Kharzeev, and A. A. Rostovtsev, The origin of thermal component in the transverse momentum spectra in high energy hadronic processes, Int. J. Mod. Phys. E 23, 1450083 (2014).

[8] R. Hagedorn, Statistical thermodynamics of strong interactions at high energies, Nuovo Cimento Suppl. 3, 147
(1965); Hadronic matter near the boiling point, Nuovo Cim. 56A, 1027 (1965).

[9] Y. I. Azimov, Y. L. Dokshitzer, V. A. Khoze, and S. I. Troyan, The string effect and QCD coherence, Phys. Lett. B 165, 147 (1985).

[10] D. E Kharzeev and K. Tuchin, From color glass condensate to quark-gluon plasma through the event horizon, Nucl. Phys. A753, 316 (2005).

[11] G. Dunne and T. Hall, QED effective action in time dependent electric backgrounds, Phys. Rev. D 58, 105022 (1998).

[12] P. Castorina, D. Kharzeev, and H. Satz, Thermal Hadronization and Hawking-Unruh radiation in QCD, Eur. Phys. J. C 52, 187 (2007).

[13] D. E. Kharzeev, E. Levin, and K. Tuchin, Multiparticle production and thermalization in high-energy QCD, Phys. Rev. C 75, 044903 (2007).

[14] L. V. Gribov, E. M. Levin, and M. G. Ryskin, Semihard processes in QCD, Phys. Rep. 100, 1 (1983).

[15] L. D. McLerran and R. Venugopalan, Computing quark and gluon distribution functions for very large nuclei, Phys. Rev. D 49, 2233 (1994). 
[16] L. D. McLerran and R. Venugopalan, Gluon distribution functions for very large nuclei at small transverse momentum, Phys. Rev. D 49, 3352 (1994).

[17] P. Jurcevic, B. P. Lanyon, P. Hauke, C. Hempel, P. Zoller, R. Blatt, and C.F. Roos, Quasiparticle engineering and entanglement propagation in a quantum many-body system, Nature (London) 511, 202 (2014).

[18] M. Rigol, V. Dunjko, V. Yurovsky, and M. Olshanii, Relaxation in a Completely Integrable Many-Body Quantum System: An $A b$ Initio Study of the Dynamics of the Highly Excited States of 1d Lattice Hard-Core Bosons, Phys. Rev. Lett. 98, 050405 (2007).

[19] Z. X. Gong and L. M. Duan, Prethermalization and dynamic phase transition in an isolated trapped ion spin chain, New J. Phys. 15, 113051 (2013).

[20] P. Calabrese and J. Cardy, Quantum quenches in extended systems, J. Stat. Mech. (2007) P06008.

[21] R. Baier, A. H. Mueller, D. Schiff, and D. T. Son, 'Bottom up' thermalization in heavy ion collisions, Phys. Lett. B 502, 51 (2001).

[22] S. Lin and E. Shuryak, Toward the AdS/CFT gravity dual for High Energy Collisions: I.Falling into the AdS, Phys. Rev. D 77, 085013 (2008).

[23] V. Balasubramanian, A. Bernamonti, J. de Boer, N. Copland, B. Craps, E. Keski-Vakkuri, B. Müller, A. Schäfer, M. Shigemori, and W. Staessens, Holographic thermalization, Phys. Rev. D 84, 026010 (2011).

[24] J. Berges, S. Floerchinger, and R. Venugopalan, Thermal excitation spectrum from entanglement in an expanding QCD string, Phys. Lett. B 778, 442 (2018).
[25] The ATLAS collaboration, Charged-particle distributions at low transverse momentum in $\sqrt{s}=13 \mathrm{TeV}$ pp interactions measured with the ATLAS detector at the LHC, Eur. Phys. J. C 76, 502 (2016); The ATLAS collaboration, Charged-particle distributions in $\sqrt{s}=13 \mathrm{TeV}$ pp interactions measured with the ATLAS detector at the LHC, Phys. Lett. B 758, 67 (2016).

[26] The ATLAS collaboration, Measurement of the exclusive $\gamma \gamma \rightarrow \mu^{+} \mu^{-}$process in proton-proton collisions at $\sqrt{S}=$ $13 \times \mathrm{TeV}$ with the ATLAS detector, Phys. Lett. B 777, 303 (2018).

[27] S. Dittmaier et al. (LHC Higgs Cross Section Working Group Collaboration), Handbook of LHC Higgs cross sections: 1. Inclusive observables, arXiv:1101.0593.

[28] The ATLAS collaboration, Measurement of inclusive and differential cross sections in the $H \rightarrow 4 l$ decay channel in pp collisions at $\sqrt{s}=13 \mathrm{TeV}$ with the ATLAS detector, J. High Energy Phys. 10 (2017) 132.

[29] Eleni Mountricha on behalf of the ATLAS collaboration, Higgs Measurements in High Resolution Channels with ATLAS, LHCP2017, Large Hadron Collider Physics Conference, Shanghai, China, June 2017, https://cds.cern .ch/record/2272996? ln=en.

[30] J. Tao on behalf of the CMS collaboration, Higgs boson measurements in high resolution channels with CMS, arXiv:1708.09215.

[31] We stress once again that we do not imply that the Higgs boson is produced thermally, but rather that its transverse momentum distribution is affected by thermal radiation. 